\title{
PARTICULARIDADES Y COMPLEJIDADES DEL TRABAJO INFANTIL: ASPECTOS CONCEPTUALES Y APROXIMACIÓN EMPÍRICA A UN FENÓMENO OCULTO CON DIMENSIONES DIVERSAS*
}

\author{
MAURICIO PADRÓN INNAMORATO"* \& PATRICIA ROMÁN REYES \\ UNIVERSIDAD NACIONAL AUTÓNOMA DE MÉXICO
}

Recibido/ Received/ Recebido: 23/08/2012 - Aceptado/ Accepted/Aprovado: 20/03/2013

\begin{abstract}
Resumen
Este artículo tiene como propósito general presentar y discutir algunos de los elementos que interrelacionados, permiten aproximarse al fenómeno del trabajo infantil. Así, no se busca exponer un panorama cuantitativo de la situación de niñas, niños y adolescentes (NNA) que trabajan, sino exhibir un marco referencial que proporcione posibles perspectivas para su abordaje, análisis e interpretación. Para lograr lo anterior se recurre a la presentación del marco jurídico, tanto internacional como en el contexto mexicano. Además, se presentan algunos datos que permiten ejemplificar la situación de NNA que trabajan. Como forma de justificar la importancia de este trabajo se debe mencionar que en México no existe un diagnóstico integral e interdisciplinario del trabajo infantil, por lo que se hace necesario profundizar en el tema por medio del diálogo entre distintas disciplinas, visiones y aproximaciones a un fenómeno de naturaleza extremadamente compleja, y que muchas veces se encuentra invisibilizado.
\end{abstract}

Palabras clave: Trabajo infantil, Conceptualización jurídica, Derechos de niñas, niños y adolescentes, México.

\section{PARTICULARITIES AND COMPLEXITIES OF CHILD LABOR: CONCEPTUAL ASPECTS AND EMPIRICAL APPROACH TO A HIDDEN PHENOMENON WITH DIFFERENT DIMENSIONS}

\begin{abstract}
This article aims to presents and discuss some elements interrelated that allow approaching to child labor phenomenon. Thus, it does not seek to expose a quantitative overview of children (boys and girls), and adolescents situation (NNA for its acronym in Spanish), but to display framework that provides possible prospects for their approach, analysis and interpretation. To achieve this, it was used the legal framework presentation, both internationally and in Mexican context, this last one does not have been frequently addressed in social science research. Also some data is presented which allows illustrating children and adolescents working situation. As a way to justify

\footnotetext{
Investigación adelantada para el Grupo de Trabajo en Mercados Laborales

* Licenciado en Trabajo social de la Universidad de la República (Uruguay), maestro en Estudios de Población de la Facultad Latinoamericana de Ciencias Sociales (México) y doctor en Estudios de Población por El Colegio de México. Investigador de Tiempo completo del Instituto de Investigaciones Jurídicas de la Universidad Nacional Autónoma de México.

Correo electrónico: mauriciopadron@gmail.com

*** Licenciada en Trabajo Social de la Universidad de la República (Uruguay), maestra Estudios de Población de la Facultad Latinoamericana de Ciencias Sociales (México) y doctora en Estudios de Población por El Colegio de México. Profesora investigadora del Centro de Investigación y Estudio Avanzados de la Población de la Universidad Autónoma del Estado de México.

Correo electrónico: promanreyes@yahoo.com.mx
} 
the importance of this work should it be mentioned that in Mexico there is no comprehensive and interdisciplinary diagnosis of child labor, so it is necessary to deepen into the issue through dialogue across disciplines, perspective and approaches to a nature phenomenon extremely complex which is often invisible.

Keywords: Child labor, Legal conceptualization, Children and adolescents rights, Mexico.

\title{
PARTICULARIDADES E COMPLEXIDADES DO TRABALHO INFANTIL: ASPECTOS CONCEITUAIS E APROXIMAÇÃO EMPÍRICA A UM FENÔMENO OCULTO CON DIVERSAS DIMENSÓES
}

\begin{abstract}
Resumo
Este artigo reflexivo tem como propósito geral apresentar e discutir alguns elementos que, inter-relacionados, permitem uma aproximação ao fenômeno do trabalho infantil. Não se procura expor um panorama quantitativo da situação de meninas, meninos e adolescentes (NNA) que trabalham, mas sim exibir um marco referencial que proporcione possíveis perspectivas para sua abordagem, sua análise e interpretação. Para conseguir isto se recorre à apresentação do marco jurídico, tanto internacional como do contexto mexicano, mesmo que pouco abordado na pesquisa em ciências sociais em geral. Além do mais, apresentam-se alguns dados que permitem exemplificar a situação de meninas, meninos e adolescentes que trabalham. Como forma de justificar a importância deste trabalho se deve mencionar que no México não existe um diagnóstico integral e interdisciplinar do trabalho infantil, pelo que se faz necessário aprofundar o tema por meio do diálogo entre diferentes disciplinas, visões e aproximações a um fenômeno de natureza extremamente complexa, e que muitas vezes se encontra invisibilizado.

Palavras chave: Trabalho infantil, Conceituação jurídica, Direitos de meninas, meninos e adolescentes, México.

Padrón, M. \& Román, P. (2013) Particularidades y complejidades del trabajo infantil: aspectos conceptuales y aproximación empírica a un fenómeno oculto con dimensiones diversas. En: Revista de la Facultad de Ciencias Económicas de la Universidad Militar Nueva Granada. rev.fac.cienc.econ, XXI (1)
\end{abstract}

JEL: J21, J82.

\section{Introducción}

Por la propia complejidad que adquiere el fenómeno en la realidad, tanto la discusión conceptual como la cuantificación de los casos de niñas, niños y adolescentes (NNA) que cotidianamente se encuentran expuestos a la necesidad u obligación de desarrollar actividades laborales se convierte en una empresa por demás difícil. Esta realidad se debe, principalmente, a que en la base del fenómeno se entrelazan factores externos, como la condición de ilegalidad de este tipo de actividades $y$, factores internos, subjetivos o de interpreta- ción de esta situación que no siempre permite identificar, distinguir y reconocer la presencia del trabajo infantil. Así nos encontramos frente a un fenómeno social complejo, multidimensional y sin lugar a dudas, que sigue estando presente en la sociedad mexicana.

A esta extendida invisibilidad de las condiciones de vida de una parte de la población infantil hace referencia por ejemplo en un informe de Amnistía Internacional donde se dice que "Históricamente la infancia es invisible (...) hasta muy recientemente no ha sido tema de interés". ${ }^{1}$ Así, en la conceptua-

1 Consulta en línea: http://www.amnistiacatalunya.org/edu/es/historia/h-precariedad.html 1 
lización del fenómeno parecen conjugarse dimensiones que tradicional $e$ incluso culturalmente no diagnosticamos, analizamos e identificamos con la profundidad que deberíamos. Complejidad que aumenta notablemente si se entiende que la población infantil desarrolla su cotidianidad (en la mayoría de los casos) dentro de las familias, es decir, en el marco de espacios privados de socialización, que imponen sus normas, valores y pautas de funcionamiento de forma intima y subjetiva.

Lo dicho conlleva a que precisar cuándo, dónde y de qué forma se desarrolla el trabajo infantil no resulte fácil, mucho menos entonces reconocerlo, analizarlo y lograr distinguir y establecer factores asociados con este fenómeno.

De ahí que surjan inquietudes tales como si se puede considerar dentro de la misma categoría de menores de edad a un niño o una niña de 5 años que a otro(a) de 12 años, que a un(a) adolescente de 17, o bajo que formas el trabajo infantil vulnera la dignidad y el desarrollo de la población infantil, o hasta donde son excluidos y por ende vulnerados.

Si bien, este trabajo no busca dar cuenta de la compleja construcción de este contexto socio cultural, si pretende aproximarse a la discusión de las posturas teóricas o conceptuales, así como describir las características básicas que asume este fenómeno y la forma en que se interrelacionan los principales elementos que hacen al trabajo infantil. Entonces, para lograr cumplir con el objetivo propuesto, se propone organizar el trabajo en tres apartados generales; en el primero se presentan las principales dimensiones y ámbitos analíticos y la forma en que se entiende el trabajo infantil principalmente desde las definiciones existentes. En un segundo apartado, se discuten los instrumentos legales de protección a NNA desarrollados e implementados a nivel internacional y nacional. En el tercer apartado, se describen brevemente algunas características de la población infantil que trabaja en México; quiénes son, en qué hogares viven y de qué forma se vinculan con las dimensiones definidas.
El documento se nutre de información documental, de referentes empíricos de entidades como el Programa de las Naciones Unidas para el Desarrollo (PNUD), la Organización Internacional del Trabajo (OIT), el Instituto Nacional de Estadística y Geografía (INEGI), el Consejo Nacional de Población (CONAPO) y de una serie de datos retomados para su procesamiento y análisis del Censo de Población y Vivienda de 2010.

Finalmente, se debe resaltar que el eje analítico $e$ interpretativo que se pretende desarrollar no busca exponer un panorama cuantitativo exhaustivo de la situación de NNA que trabajan, sino exhibir un marco referencial que permita desarrollar y presentar perspectivas válidas para reconocer y entender las condiciones sociales, económicas y culturales (es decir, materiales y simbólicas) que subyacen tanto como causas y consecuencias del trabajo de NNA.

\section{Aproximaciones conceptuales: delimitación del fenómeno y de las dimensiones de análisis}

Una de las primeras dificultades a la que se enfrentan los investigadores al abordar el fenómeno del trabajo infantil, radica precisamente en la definición de las actividades que caen dentro del supuesto de trabajo, y que hace referencia a esta población específica. Es claro que ciertos tipos de tareas que realizan niñas y niños encuadran perfectamente en el trabajo que debe ser erradicado, entre ellas se encuentran las que la OIT define en el Convenio 182 como "Las peores formas de trabajo infantil" 2 .

En este convenio se reconoce en el artículo 1 como "niño" a toda persona menor de 18 años de edad y se consideran en el artículo 3 como peores formas de trabajo infantil las siguientes: a) todas las formas de esclavitud o las prácticas análogas a la esclavitud, como la venta y la trata de niños, la servidumbre por deudas y la condición de siervo, y el trabajo forzoso $\mathrm{u}$ obligatorio, incluido el reclutamiento forzoso u obligatorio de niños para utilizarlos en conflictos armados; b) la utilización, el reclutamiento o la oferta de niños para la prostitución, la producción de porno-

\footnotetext{
2 Algunos especialistas incluso se niegan a esta definición, y sostienen que este tipo de actividades ni siquiera pueden considerarse "trabajo", dada su naturaleza. Una alternativa es identificarlas como "prácticas intolerables en contra de niñas y niños".
} 
grafía o actuaciones pornográficas; c) la utilización, el reclutamiento o la oferta de niños para la realización de actividades ilícitas, en particular la producción y el tráfico de estupefacientes, tal como se definen en los tratados internacionales pertinentes, y d) el trabajo que, por su naturaleza o por las condiciones en que se lleva a cabo, es probable que dañe la salud, la seguridad o la moralidad de los niños ${ }^{3}$.

Dada la clasificación anterior, se puede decir que en el otro extremo se podrían identificar las situaciones de ausencia de cualquier forma de explotación económica, y por lo tanto, se estaría en presencia de una garantía plena de los derechos de niñas, niños y adolescentes. Sin embargo, en el medio existen una serie de situaciones difíciles de catalogar, ya que si bien no constituyen una forma evidente de explotación, pueden afectar seriamente el desarrollo de esta población específica (Padrón \& González, 2012).

Pero este escenario se complejiza cuando existen prácticas culturales en las que ciertas actividades forman parte de la transmisión de valores, especialmente familiares y comunitarias, hacia las nuevas generaciones. En el caso de Latinoamérica, de acuerdo con Salazar (1996, 180), "los niños trabajan en general, porque su familia es pobre, pero también por factores culturales. La concepción que subyace de fondo parece ser la de que todos los miembros de la familia son proveedores económicos de la misma y a través del ejercicio de esta responsabilidad se forma a los niños de hoy para ser los adultos competentes del mañana. En todos los países se encontraron formas de producción en las que la familia actúa como bloque, dándose por sobreentendida la colaboración activa de los niños. Los padres justifican la vinculación de sus hijos al trabajo aduciendo que en él adquieren valores como la responsabilidad, la autonomía y la tenacidad para sobrellevar las dificultades o para soportar sacrificios. Además se ve el trabajo como una protección contra los vicios y el ocio que conduce a la delincuencia”.

Ahora, la OIT define al trabajo infantil como "toda actividad económica llevada a cabo por personas menores de 15 años de edad, sin importar el estatus ocupacional (trabajo asalariado, trabajo independiente, trabajo familiar no remunerado, etc.). Ello no incluye los quehaceres del hogar realizados en su propio hogar, excepto donde los quehaceres del hogar puedan ser considerados una actividad económica-como, por ejemplo, cuando un niño dedica todo su tiempo a estos quehaceres para que sus padres puedan trabajar fuera del hogar y ello signifique privarlo de la posibilidad de ir a la escuela" (OIT, 1996, 5).

Aunque lo dicho, muestra que los instrumentos jurídicos son de gran utilidad para definir el trabajo infantil, también se debe reconocer que resultan insuficientes en la medida en que no consideran contextos concretos, ni situaciones que caen dentro de lo que se denomina "zona de penumbra", o aquellos casos que se configuran como "casos difíciles" 4 , es decir, que no pueden identificarse claramente como trabajo de acuerdo con las definiciones legales, pero que constituyen actividades que dificultan o imposibilitan claramente el ejercicio de otros derechos de la niña o niño (Padrón \& González, 2012).

Como se dijo antes, el hecho de que en las definiciones legales juega un papel poco relevante el contexto, agrega un nuevo factor de complejidad a la hora de medir un fenómeno ya de por si difícil. En este sentido, se cree que para dar una adecuada respuesta jurídica sería necesario no sólo considerar los factores que podrían calificarse como "objetivos", tales como son el horario o el tipo de actividad, sino también considerar las razones por las que niñas y niños desempeña cierta actividad; por lo que ésta aproximación implicaría considerar de manera integral factores sociales, económicos, culturales.

De lo planteado hasta ahora, se puede derivar la idea de que una adecuada comprensión del fenómeno del trabajo infantil es fundamental para erradicar

\footnotetext{
Véase: http://www.ilo.org/public/spanish/standards/relm/ilc/ilc87/com-chic.htm

La distinción entre casos fáciles y casos difíciles ha sido utilizado en la teoría y filosofía del derecho para diferenciar aquellos casos que pueden ser resueltos con la simple aplicación de la norma, de aquellos que requieren de la interpretación judicial: "Casos fáciles son aquellos en los que no hay más que aplicación pura y simple del derecho, mientras que en los casos difíciles la cuestión en litigio no está determinada en los estándares jurídicos existentes y, por lo tanto, se requiere de una labor interpretativa” (Vázquez, 2007, 206).
} 
aquellas prácticas que son nocivas para la infancia y adolescencia. Y como se vio, no necesariamente son los instrumentos jurídicos o normativos los que nos pueden dar claridad en este aspecto. La perspectiva a asumir implicaría considerar el problema como un proceso complejo, respecto del que es necesario un enfoque interdisciplinario, debido a que el derecho por sí mismo no ha conseguido dar una respuesta suficiente.

\section{Revisión de los instrumentos legales para la protección de la infancia}

En repetidas ocasiones los temas vinculados con la titularidad y garantía de los derechos de niñas y niños han sido abordados de una manera simplista e inadecuada. Es evidente que las leyes son necesarias pero no suficientes para cambiar una situación, y en el caso de los derechos de niñas y niños esta realidad es patente, debido a los obstáculos que su incorporación como titulares plenos de derechos ha tenido tanto en el ámbito teórico como práctico. A más de 20 años de que la Convención sobre los Derechos del Niño fuera aprobada por la Asamblea General de Naciones Unidas, los avances son limitados, particularmente en algunos aspectos (Padrón \& González, 2012).

Uno de los ámbitos en el que se observan grandes dificultades tiene que ver precisamente con el trabajo infantil, pues la legislación, como se ha dicho ya, no ha logrado conseguir su erradicación. En este sentido, la Convención establece en su artículo 32 la obligación de los Estados de proteger al niño de toda forma de explotación o trabajo perjudicial y de garantizar esto mediante varios medios:

\section{"Artículo 32.}

1. Los Estados partes reconocen el derecho del niño a estar protegido contra la explotación económica y contra el desempeño de cualquier trabajo que pueda ser peligroso o entorpecer su educación, o que sea nocivo para su salud o para su desarrollo físico, mental, espiritual o social.

2. Los Estados partes adoptarán medidas legislativas, administrativas, sociales y educacionales para garantizar la aplicación del presente artículo. Con ese propósito y teniendo en cuenta las disposiciones pertinentes de otros instrumentos internacionales, los Estados partes, en particular:

a) Fijarán una edad o edades mínimas para trabajar;

b) Dispondrán la reglamentación apropiada de los horarios y condiciones de trabajo; $y$

c) Estipularán las penalidades y otras sanciones apropiadas para asegurar la aplicación efectiva del presente artículo."

En este artículo pueden distinguirse dos partes: en primer término un derecho a ser protegido en contra la explotación económica y del desempeño de cualquier trabajo nocivo para la salud, educación o desarrollo y, en segundo lugar, una obligación para los Estados Partes de fijar edades mínimas y reglas sobre las condiciones del trabajo (González, 2008, 456). Sin embargo, en la realidad no resulta sencillo ni la protección en contra del trabajo peligroso ni el cumplimiento de la obligación de fijar una edad mínima, pues si ésta no va acompañada de otro tipo de acciones puede incluso desencadenar lo que la primera parte de la disposición pretende impedir, es decir, generar condiciones de mayor vulnerabilidad en el desempeño de trabajo de ciertas actividades para las personas menores de edad.

Así, una de las mayores dificultades para el cumplimiento efectivo de lo planteado por la Convención en los países en vías de desarrollo, como es el caso de México, es la falta de un Estado de bienestar que garantice los derechos económicos, sociales y culturales. A esto se debe sumar las profundas desigualdades económicas y sociales, y las condiciones de pobreza de muchas familias, situaciones que se consideran como determinantes de que niñas y niños tengan que realizar actividades consideradas como trabajo para contribuir con los ingresos de su grupo familiar.

A lo anterior hay que agregar, como se decía antes, la pluralidad cultural que es característica de los países latinoamericanos, que incide en las formas de entender la interacción social que no siempre es compatible con una comprensión rígida de la Convención sobre los Derechos del Niño en lo que respecta a la prohibición del trabajo infantil. 
Para el caso mexicano, el marco jurídico del trabajo infantil está integrado por el artículo 123 de la Constitución, específicamente en las fracciones II, III y XI, así como por la ley reglamentaria de dicho artículo, la Ley Federal del Trabajo en sus artículos 22, 23, 173 a 180, 362 , 372, 995. Desde 1917 se contempló en la Constitución mexicana ${ }^{5}$ la prohibición del trabajo infantil ${ }^{6}$.

El texto original incluía en esta prohibición a todos los menores de 12 años y establecía restricciones para el trabajo de los mayores de 12 años y menores de 16 años. En 1962 el texto fue reformado tanto para elevar la edad de prohibición a los 14 años como para modificar la redacción que aludía a la imposibilidad de que su trabajo fuera objeto de contrato (Kurczyn, 2006, 410).

"Artículo 123. Toda persona tiene derecho al trabajo digno y socialmente útil; al efecto, se promoverán la creación de empleos y la organización social de trabajo, conforme a la ley. El Congreso de la Unión, sin contravenir a las bases siguientes deberá expedir leyes sobre el trabajo, las cuales regirán:

II. La jornada máxima de trabajo nocturno será de 7 horas. Quedan prohibidas: las labores insalubres o peligrosas, el trabajo nocturno industrial y todo otro trabajo después de las diez de la noche, de los menores de dieciséis años;

III. Queda prohibida la utilización del trabajo de los menores de catorce años. Los mayores de esta edad y menores de dieciséis tendrán como jornada máxima la de seis horas.
XI. Cuando, por circunstancias extraordinarias deban aumentarse las horas de jornada, se abonará como salario por el tiempo excedente un 100\% más de lo fijado para las horas normales. En ningún caso el trabajo extraordinario podrá exceder de tres horas diarias, ni de tres veces consecutivas. Los menores de dieciséis años no serán admitidos en esta clase de trabajos (...)".

Como puede advertirse, el texto constitucional contempla dos supuestos vinculados al trabajo infantil: la prohibición del trabajo de los menores de 14 años y la restricción de la jornada para los menores de 16. El constituyente, tanto en la redacción original como en la reforma, no estableció una prohibición absoluta del trabajo a los adolescentes, sino que formuló una limitación directa o indirecta de determinada actividades. Lo anterior tiene como finalidad tanto proteger el derecho de niñas y niños a la integridad física y mental por parte de los ascendientes, tutores y custodios, como regular el trabajo (Kurczyn, 2006,408).

Así, dentro del período comprendido de 0 a 18 años que contempla la Convención en la definición de niño, en el caso de México podemos distinguir tres franjas etarias con regulaciones distintas:

- De 0 a 13 años: respecto de los cuales hay una prohibición absoluta de trabajar.

- De 14 y 15 años: los integrantes de este grupo, a quienes podemos identificar como "adolescentes trabajadores", puede ser contratado con ciertas restricciones.

5 La actual Constitución mexicana fue promulgada el 5 de febrero de 1917 por Venustiano Carranza, Primer Jefe del Ejército Constitucionalista y Encargado del Poder Ejecutivo de los Estados Unidos Mexicanos. En ese momento histórico México salía de la Revolución Mexicana, una guerra civil iniciada en 1910.

$6 \quad$ Art. 123.- El Congreso de la Unión y las Legislaturas de los Estados deberán expedir leyes sobre el trabajo, fundadas en las necesidades de cada región, sin contravenir a las bases siguientes, las cuales regirán el trabajo de los obreros, jornaleros, empleados, domésticos y artesanos, y de una manera general todo contrato de trabajo:

II.- La jornada máxima de trabajo nocturno será de siete horas. Quedan prohibidas las labores insalubres o peligrosas para las mujeres en general y para los jóvenes menores de diez y seis años. Queda también prohibido a unas y otros el trabajo nocturno industrial; y en los establecimientos comerciales no podrán trabajar después de las diez de la noche.

III.- Los jóvenes mayores de doce años y menores de dieciséis, tendrán como jornada máxima la de seis horas. El trabajo de los niños menores de doce años no podrá ser objeto de contrato.

XI.- Cuando por circunstancias extraordinarias deban aumentarse las horas de jornada, se abonará como salario por el tiempo excedente, un ciento por ciento más de lo fijado para las horas normales. En ningún caso el trabajo extraordinario podrá exceder de tres horas diarias, ni de tres veces consecutivas. Los hombres menores de diez y seis años y las mujeres de cualquiera edad, no serán admitidos en esta clase de trabajos. 
- De 16 y 17 años: para efectos laborales quienes se encuentran dentro de este rango son considerados como mayores de edad, con algunas excepciones contempladas en la Ley $\mathrm{Fe}^{-}$ deral del Trabajo.

La norma constitucional se complementa con lo establecido en la Ley Federal del Trabajo respecto de las personas menores de 16 años. El Título Quinto Bis: Trabajo de los menores contiene una serie de disposiciones que contemplan un régimen especial para este grupo, con prerrogativas y requerimientos concretos. En este sentido, el artículo 174 obliga a los patrones a exigir un certificado médico que acredite aptitud para el trabajo. Se restringe también la intervención de adolescentes en ciertas actividades como en el expendio de bebidas embriagantes, labores que afecten su moral o buenas costumbres, trabajos ambulantes, subterráneos, submarinos, peligrosos o insalubres, superiores a sus fuerzas o en establecimientos industriales después de las diez de la noche (art. 175 y 176). En el mismo título se limita la jornada laboral, que no podrá exceder de 6 horas diarias divididas en períodos de 3 horas máximo con un descanso de una hora (art. 177). El período vacacional deberá ser al menos de 18 días al año (art. 178). Además, el trabajo de las personas de este grupo etario está sometido también a la autorización de los padres o tutores o, en su caso, de la Junta de Conciliación y Arbitraje, del Inspector del Trabajo o de la Autoridad Política (art. 23), así como a haber concluido la educación obligatoria (art. 22). La ley contempla también algunas restricciones a los derechos de los adolescentes trabajadores, pues tienen impedimento para formar parte de las directivas de los sindicatos (art. 372) (Kurczyn, 2006, 410-411).

La sanción por la violación a las normas relativas al trabajo de los menores de 16 años consiste en la imposición de una multa por el equivalente de 3 a 155 veces el salario mínimo general, según lo establece el artículo 995. La supervisión corresponde a la Secretaría del Trabajo y Previsión Social para las empresas o establecimientos de jurisdicción federal en tanto que para las empresas o establecimien- tos de jurisdicción local dicho control se realiza de acuerdo con las administraciones de cada uno de los gobiernos locales (Kurczyn, 2006, 411).

Estas consideraciones, sumadas a la prohibición constitucional referida en la misma fracción II de realizar trabajos peligrosos o insalubres y nocturnos así como jornadas extraordinarias, integran el marco legal de protección a los derechos de niñas y niños en contra de cualquier tipo de explotación económica. A éste se suma, desde luego, la Convención sobre los Derechos del Niño y el Convenio sobre la prohibición de las peores formas de trabajo infantil y la acción inmediata para su eliminación (Convenio 182 de la OIT), ambos ratificados por el Estado mexicano.

En este punto es indispensable mencionar que México aún no ha ratificado el Convenio sobre la edad mínima de admisión al empleo de la OIT (Convenio 1387), lo cual debilita el sistema de protección para niñas y niños en contra del trabajo y la explotación infantil.

Como puede advertirse, sin dejar de reconocer algunos puntos débiles y otros aspectos discutibles, existe una adecuada regulación en México desde el punto de vista formal respecto del trabajo infantil y adolescente. Sin embargo, como se verá a continuación, la legislación no ha tenido los efectos deseados, pues las cifras muestran que, a pesar de la prohibición del trabajo de las personas menores de 14 años, niñas y niños siguen desempeñando actividades económicas.

Así lo reconoce el Comité de los derechos del niño en las Observaciones Finales al Informe presentado por México (CRC/C/MEX/CO/3) de 8 de junio de 2006 en el rubro "Explotación económica":

"62. Al tomar nota de las actividades emprendidas por el Estado Parte para reducir el trabajo infantil y de la reducción del número de niños que trabajan en el país, el Comité expresa su preocupación por el trabajo infantil generaliza-

7 En este Convenio la edad mínima está fijada en los 15 años, en el caso mexicano la edad mínima queda establecida en los 14 años de edad. 
do, en particular entre los niños indígenas, y por la insuficiencia de las políticas basadas en los derechos para proteger los derechos de los niños y adolescentes que trabajan. Al Comité le preocupa en particular el gran número de niños que realizan trabajos domésticos y que son vulnerables a los abusos.

63. El Comité exhorta al Estado a que intensifique sus medidas de lucha contra el trabajo infantil. Recomienda que el Estado:

a) Elabore, de manera participativa, una estrategia y un plan de acción para reducir el trabajo infantil y salvaguardar los derechos de los niños que trabajan;

b) Fortalezca la Inspección del Trabajo a fin de asegurar la aplicación eficaz de las leyes relativas al trabajo infantil, en particular la prohibición del empleo de niños que no han alcanzado la edad mínima para trabajar;

c) Ratifique el Convenio de la OIT № 138 sobre la edad mínima de admisión al empleo (1973);

d) Solicite la asistencia de la OIT/IPEC a este respecto."

\section{La Ley Federal del Trabajo mexicana}

La Ley Federal del Trabajo publicada en el Diario Oficial de la Federación el 1 de abril de 1970, última reforma publicada el 30 de noviembre de 2012, establece varias disposiciones para la protección de las niñas, niños y adolescentes. Así, por ejemplo establece que no producirá efecto legal ni impedirá el goce y el ejercicio de los derechos, sea escrita o verbal, la estipulación que establezca: trabajos para niños menores de catorce años; horas extraordinarias de trabajo para los menores de dieciséis años, y trabajo nocturno industrial o el trabajo después de las veintidós horas, para menores de dieciséis años (artículo 5).

Se prohíbe la utilización del trabajo de los menores de catorce años, y también de los mayores de catorce años y menores de dieciséis que no hayan terminado su educación obligatoria, salvo los casos de excepción que apruebe la autoridad correspondiente en que a su juicio haya compatibilidad entre los estudios y el trabajo (artículo 22). En cuanto a los mayores de dieciséis años, años pueden prestar libremente sus servicios, con las limitaciones establecidas en esta Ley. Sin embargo, necesitan autorización de sus padres o tutores y a falta de ellos, del sindicato a que pertenezcan, de la Junta de Conciliación y Arbitraje, del Inspector del Trabajo o de la Autoridad Política (artículo 23).

Se prohíbe: la utilización de menores de dieciocho años para la prestación de servicios fuera de la República, salvo que se trate de técnicos, profesionales, artistas, deportistas y, en general, de trabajadores especializados (artículo 29); la utilización del trabajo de los menores de dieciséis años, en: a) expendios de bebidas embriagantes de consumo inmediato, b) trabajos susceptibles de afectar su moralidad o sus buenas costumbres, trabajos ambulantes, salvo autorización especial de la inspección de trabajo, d) trabajos subterráneos o submarinos, e) labores peligrosas o insalubres, f) trabajos superiores a sus fuerzas y los que puedan impedir o retardar su desarrollo físico normal, g) establecimientos no industriales después de las diez de la noche; la utilización del trabajo de los menores de dieciocho años en trabajos nocturnos industriales (artículo 175); la utilización del trabajo de los menores de dieciséis años en horas extraordinarias y en los días domingos y de descanso obligatorio. En caso de violación de esta prohibición, las horas extraordinarias se pagarán con un doscientos por ciento más del salario que corresponda a las horas de la jornada, y el salario de los días domingos y de descanso obligatorio, de conformidad con lo dispuesto en los artículos 73 y 75 (artículo 178); el trabajo de menores de quince años en buques y el trabajo de menores de dieciocho en calidad de pañoleros o fogoneros (artículo 191), y el trabajo de los menores de dieciséis años en maniobras de servicio público en zonas bajo jurisdicción federal (artículo 267). Además, se establece que el Reglamento contendrá, entre otros, los labores insalubres y peligrosas que no deben desempeñar los menores y la protección que deben tener (artículo 423).

Los patrones que tengan a su servicio menores de dieciséis años están obligados a: exigir que se les exhiban los certificados médicos que acrediten que están aptos para el trabajo; llevar un registro de 
inspección especial, con indicación de la fecha de su nacimiento, clase de trabajo, horario, salario y demás condiciones generales de trabajo; distribuir el trabajo a fin de que dispongan del tiempo necesario para cumplir sus programas escolares; proporcionarles capacitación y adiestramiento en los términos de esta Ley, y proporcionar a las autoridades del trabajo los informes que soliciten (artículo 180).

Los mayores de catorce y menores de dieciséis años deberán obtener un certificado médico que acredite su aptitud para el trabajo y someterse a los exámenes médicos que ordene la Inspección del Trabajo para que un patrón pueda utilizar sus servicios (artículo 174).

La jornada de trabajo de los menores de dieciséis años no debe exceder de seis horas diarias y debe dividirse en períodos máximos de tres horas, con reposos de una hora por lo menos, entre las distintas jornadas (artículo 177). En cuanto a las vacaciones, los menores de dieciséis años disfrutarán de un periodo anual de vacaciones pagadas de dieciocho días laborables, por lo menos (artículo 179).

El trabajo de los mayores de catorce años y menores de dieciséis queda sujeto a vigilancia y protección especiales de la Inspección del Trabajo (artículo 173). Entre los deberes y atribuciones de los Inspectores del Trabajo esta el vigilar el cumplimiento de las normas de trabajo, especialmente de las que establecen los derechos y obligaciones de trabajadores y patrones, de las que reglamentan el trabajo de las mujeres y los menores, y de las que de-terminan las medidas preventivas de riesgos de trabajo, seguridad e higiene (artículo 541).

El cumplimiento de estas disposiciones esta a cargo de la Secretaría de Trabajo y Pre-visión Social. Así, el Reglamento Interior de la Secretaría del Trabajo y Previsión Social publicado en el Diario Oficial de la Federación el 14 de noviembre de 2008, establece en su artículo 18, que corresponde a la Dirección General de Inspección Federal del Trabajo de la Secretaría de Trabajo y Previsión Social, proteger y vigilar, en el ámbito de competencia de la autoridad federal, el trabajo de los mayores de catorce y menores de dieciséis años y el cumplimien- to de las restricciones del trabajo de los mayores de dieciséis y menores de dieciocho años; supervisar que las delegaciones federales del trabajo expidan las autorizaciones a los menores trabajadores que señala la Ley Federal del Trabajo, verifiquen que cuenten con sus certificados médicos de aptitud para el trabajo, y ordenen se les practiquen los exámenes médicos a que deben someterse; y que a las delegaciones federales del trabajo les corresponde vigilar el cumplimiento de las disposiciones legales en materia del trabajo de mayores de catorce y menores de dieciséis años, de las restricciones de los mayores de dieciséis y menores de dieciocho años; de las normas que reglamentan el trabajo de mujeres en estado de gestación o periodo de lactancia, así como la oportuna integración y funcionamiento de las comisiones de seguridad e higiene y mixtas de capacitación y adiestramiento en los centros de trabajo de las ramas sujetas a la competencia federal o con el auxilio de las autoridades estatales del trabajo en aquellas sujetas a la competencia local (artículo 33).

\section{Niños, niñas y adolescentes como sujetos de derechos}

De acuerdo con Barrientos \& Corvalán (1996), es importante tener en cuenta que en cierta forma la infancia a lo largo de la historia se ha estudiado desde la perspectiva cultural de la modernidad, ya que social, cultural, política, jurídica e incluso económicamente, esta población (como sujetos históricos) han formado parte (y continúan formando parte) de los principales grupos vulnerables y excluidos socialmente. En cierta medida, esto obedece a que la construcción del contexto socio cultural de NNA (su vida en familia, su inserción en los procesos sociales, educativos, laborales) sea un proceso apenas percibido en el estudio de las ciencias sociales.

Ahora, los avances realmente importantes, el gran salto cualitativo en relación a la forma de entender, analizar y ubicar en el contexto social más general a la población infantil, se empieza a producir muy recientemente (a partir del siglo $\mathrm{XX}$ ), con la Declaración Universal de los Derechos Humanos de manera extensiva, con la Declaración Universal 
de los Derechos del Niño y, sobre todo de manera más concreta, con la Convención sobre los Derechos del Niño. ${ }^{8}$

Sin embargo como se dijo antes, el establecimiento formal por medio de las normas no garantiza la efectividad o el ejercicio de los derechos, y en este sentido es la misma Convención la que establece de manera expresa que el cumplimiento de los derechos más básicos como la salud y la educación, dependerán de la situación social, económica y política del país, así como de las condiciones de las familias a las que pertenecen los niños. Pero más allá de esta observación explícita, se debe reconocer que este instrumento ha permitido ir modificando la manera de conceptualizar a los niños y niñas, y así asumirlos como sujetos de derechos, posicionándoles en un lugar distinto en la sociedad, donde empiezan a perder su condición de invisibilidad.

A pesar de estos cambios, la idea básica que sigue permeando a estos conceptos es que NNA no han alcanzado un "completo desarrollo". Así, la postura histórica de acuerdo con González $(2011,3)$, que consideraba al niño como un adulto en miniatura sigue estando presente en muchas de las posiciones, al caracterizarlos o describirlos con base en lo que no tienen en comparación con los mayores, y no partiendo de las diferencias en las estructuras físicas, mentales, etcétera. De ahí que esta autora proponga construir una fundamentación de los derechos de NNA que parta de sus cualidades como tales, es decir, adecuada a los rasgos que le distinguen y de las necesidades que le son propias.

Como se advierte, la condición de "menor de edad" coloca a los niños en una situación de constante dependencia frente a los adultos, lo que en realidad les impide el ejercicio de ciertos derechos humanos; o condiciona el ejercicio de algunos derechos por medio de la necesidad de que los acompañe una persona con más conocimiento del problema (nótese que no necesariamente debe ser un "mayor de edad”) (González, 2011).
Durante siglos el "niño" fue considerado únicamente un elemento de renovación de la estructura social, su valor estaba determinado por su pertenencia a una familia y como tal se lo definió en relación a, o como parte de, la esfera paterna (familia de pertenencia).

Como se ha mencionado líneas arriba, no se puede dejar de reconocer que las concepciones de la infancia han cambiado considerablemente a lo largo de la historia. Estos cambios en la noción de la infancia tienen que ver con los modos de organización socioeconómica de las sociedades, con las formas o pautas de crianza, con los intereses sociopolíticos, con el desarrollo de las teorías pedagógicas así como con el reconocimiento de los derechos de la infancia en las sociedades occidentales y con el desarrollo de políticas sociales al respecto. Por todo ello la infancia, más que una realidad social objetiva y universal, es ante todo un consenso social (Alzate, 2002). Pero la dificultad que se ha enfrentado para lograr ese consenso se basa, en gran medida, en las complejidades que tiene el análisis de esta población.

Por esto último, realizar un estudio acerca de la población infantil implica reconocer que se abordan unidades de una compleja heterogeneidad. En países como México, marcado por profundas desigualdades sociales, económicas y regionales, las condiciones de desarrollo son diversas. Del mismo modo, si bien el análisis se centra en un grupo poblacional de un rango de edad determinado, se debe partir del supuesto de que es un grupo que asume una amplia diversidad de prácticas, visiones y valores, que determinan que la heterogeneidad sea una característica importante del grupo conformado por NNA. Así, como señala Alzate $(2012,13)$ :
"La antigua sociedad tradicional occiden- tal no podía representarse bien al niño y menos aún al adolescente; la duración de la infancia se reducía al período de su ma- yor fragilidad, cuando la cría del hombre no puede valerse por sí misma; en cuan-

8 En 1989 se firma en las Naciones Unidas la Convención Internacional sobre los Derechos del Niño, la cual representa un instrumento muy avanzado en términos de contenido, de fuerza vinculante y de impacto cultural para la defensa de NNA. México ratifica esta Convención en 1990, y a partir de esta ratificación se considera niño a todo ser humano menor de 18 años de edad. 
to podía desenvolverse físicamente, se le mezclaba rápidamente con los adultos, con quienes compartía trabajos y juegos. El bebé se convertía en seguida en un hombre joven sin pasar por las etapas de la juventud".

El trabajo infantil suele ser definido como el trabajo que priva a los niños y las niñas de su infancia, su potencial (interfiere en su escolarización) y su dignidad, y que es nocivo para su desarrollo físico (dañino) y mental (social o moralmente perjudicial) ${ }^{9}$. Si bien consensualmente todos condenamos las formas de trabajo que vulneran las condiciones de vida de la población infantil, también tenemos que reconocer que no todo el trabajo efectuado por niños $y$ niñas tendrá un impacto negativo y condenatorio. Veamos ahora algunos datos sobre la situación de la población NNA en México y sus condiciones y formas de trabajo.

\section{Circunstancias y contexto para el trabajo infantil}

En México de acuerdo con cifras del Instituto Nacional de Estadística y Geografía (INEGI, 2013) hay poco más de 3 millones de niños y niñas entre $5 \mathrm{y}$ 17 años de edad que trabajan (3.014.800), esta situación no se constituye en todos los casos como una opción de vida, ya que para muchas de estas personas es una forma de sobrevivencia. De este modo el trabajo infantil se concibe como una manifestación clara de la pobreza, vulnerabilidad, violencia y exclusión de una gran cantidad de niños y niñas.

De acuerdo como se ha planteado en este trabajo el tema del trabajo infantil, es importante señalar que buena parte de las opiniones sobre el asunto coinciden en desmitificarlo como un hecho contraproducente siempre que ese trabajo no interfiera con las actividades que los niños deberían estar haciendo con respecto a su educación, recreación, descanso y cuidado físico de su persona.
En este sentido se plantea que el trabajo infantil responde a necesidades, circunstancias y contextos muy diversos, que deben ser considerados. Esta posición permite repensar los estereotipos del trabajo infantil como una situación dañina y perjudicial, sino que además posibilita consideran como valiosas estas actividades laborales, en determinadas circunstancias.

De esta forma en la discusión de las modalidades que adquiere el trabajo infantil entra en juego un componente de tipo cultural, porque en muchas comunidades el trabajo (enfatizando que para que sea aceptado tiene que excluir siempre un riesgo de maltrato físico, es decir no es aceptable por ejemplo el trabajo de un niño y una mina) forma parte de la educación que los padres o la comunidad da a los niños, e implica la formación en la responsabilidad en ciertos valores como en la autonomía y la autosuficiencia.

Es imprescindible que ante la necesidad de desarrollar actividades laborales dadas las precarias condiciones de vida de gran parte de la población, estas actividades se llevaran a cabo en situaciones legalmente reguladas que eviten al máximo las condiciones de explotación del trabajo de los menores, y que no afecten las posibilidades de los niños y las niñas de continuar sus estudios, de jugar, de tener tiempo libre. En este punto es importante señalar que el hecho de reconocer esta realidad no implica legalizarla, por lo que se debe controlar al máximo la explotación de los menores (Maccise, 2006).

\section{Algunas características de las niñas, niños y adolescentes en México}

Para comenzar a dibujar el perfil de la población NNA que trabaja en México, primero es necesario comentar brevemente que uno de los problemas vinculados al fenómeno del trabajo infantil en México había sido, hasta hace muy poco tiempo, la falta de cifras que permitieran conocer las dimensiones y características del problema. Así lo había señalado

9 De acuerdo con la definición de la Organización Internacional del Trabajo (OIT) disponible en: http://white.oit.org.pe/ipec/pagina. php?seccion=6. 
ya el Comité de los Derechos del Niño en las observaciones al Estado mexicano realizadas en 2006. Finalmente, en el 2007 se incluyó en la Encuesta Nacional de Ocupación y Empleo (ENOE), el Módulo sobre Trabajo Infantil (MTI), mismo que se replicó en el 2009. De manera general se puede decir que la información recopilada mediante este instrumento permite una aproximación a las características sociodemográficas de las niñas, niños y adolescentes de 5 a 17 años y que realizan actividades económicas, domésticas y escolares en el país.

La encuesta (INEGI, 2009) define a la población de referencia a partir del concepto de "niño" adoptado por UNICEF, con base en el Convenio 182 de la OIT, entendiéndolo como toda persona menor de 18 años. Así, el límite superior establecido son los 17 años cumplidos y el límite de edad inferior se definió tomando como referencia las experiencias internacionales de recolección de datos sobre trabajo infantil, donde de manera general se toman los 5 años de edad.

Bajo estos criterios, la población de estudio del MTI 2009 quedó definida como las personas de 5 a 17 años de edad, que residen de manera habitual en las viviendas particulares que forman parte de la muestra de la ENOE del cuarto trimestre del 2009, a las que se le aplicaron los cuestionarios sobre las actividades de niños, niñas y adolescentes.

En cuanto a la cobertura geográfica del MTI (INEGI, 2013), se puede decir que el módulo fue aplicado en la muestra de viviendas particulares de la ENOE con presencia de población infantil de 5 a 17 años, por lo que, partiendo del diseño estadístico de la encuesta, el número de viviendas y personas seleccionadas es suficiente para ofrecer información sobre el número de niños y niñas que realizan actividades económicas y domésticas a nivel nacional, para las áreas urbanas, para las menos urbanizadas y a nivel de entidades federativas.

En cuanto a la definición de trabajo (INEGI, 2013), éste es conceptualizado de manera amplia y se engloban en el término las actividades económicas destinadas a la producción de bienes y servicios para el mercado, así como las actividades domésticas no remuneradas orientadas a la producción de bienes y servicios para el consumo de los miembros del hogar.

Así, con base en el enfoque de la fuerza de trabajo, es decir, teniendo en cuenta la mano de obra, y considerando la semana anterior al levantamiento de la información (período de referencia), la encuesta define como población ocupada a las personas de 5 a 17 años cumplidos que efectivamente realizaron actividades económicas. Cabe aclarar que, en el marco de las mediciones sobre trabajo infantil, la categoría de "ocupado" refiere a aquellas personas que en el período de referencia realizaban alguna actividad económica o que se incorporaron a una durante la semana en que se realiza la medición.

Es necesario recordar que, el trabajo con datos provenientes de fuentes de información secundarias tiene sus limitaciones, una de ellas es que no siempre se pueden hacer aproximaciones empíricas partiendo de las definiciones teóricas deseadas. Es decir, los datos existentes y disponibles levantados por terceros parten de conceptualizaciones propias que no necesariamente son acordes con las manejadas por los usuarios de la información, situación que pone ciertas restricciones al análisis (Padrón, 2012).

Esbozar el perfil de los hogares con NNA en el México actual tiene la utilidad de contribuir como un instrumento diagnóstico a partir del cual discutir las repercusiones que tienen las características de los hogares de origen en las condiciones de vida y estudio de esta población, en el entendido que es indispensable identificar las peculiaridades de los hogares, tanto para el diseño de políticas públicas, como para el avance de la investigación académica relacionada con la situación de los niños en el país. Contextualizar estos cambios y tendencias permite observar el hogar como una entidad intermedia entre los niños y las niñas y el contexto social en que se ubican, donde el hogar actúa como un eje articulador de lo individual y lo social (Padrón \& Román, 2010).

De acuerdo con datos del Censo de Población y Vivienda 2010 (INEGI, 2013) del total de población residente en el país en ese año 34,9\% eran niños 
y niñas, es decir que, de las 112 millones de personas que conformaban la población del país, 39.2 millones tenían menos de 18 años. Así, en México en el año 2010 poco más de 1 de cada tres habitantes era menor de 18 años, situación que ubica a la población infantil como uno de los grupos de población más importante, tanto por el volumen que concentra como por los requerimientos específicos que plantea en ámbitos tan importantes como salud, educación y seguridad social, entre otros (Padrón \& Román, 2010).

Sin embargo, y a pesar de este relevancia numérica, la población infantil a partir del año 2000 inició una lenta pero constante disminución al pasar de representar el 39,7\% de la población total a 34,9\% para el año 2010, y se estima que en el año 2020 esta población ronde el $25 \%$ de la población total nacional; es decir, cerca de una cuarta parte de la población nacional. De acuerdo con el promedio nacional este grupo de edad es el único en el que el total de hombres supera al de mujeres, en este sentido se aprecia de acuerdo con las datos manejados, que la diferencia total es de 524 mil varones, la cual se traduce en términos porcentuales en 50,7\% de niños y 49,3\% de niñas (Padrón \& Román, 2010).

De acuerdo con cifras del Consejo Nacional de Evaluación de la Política de Desarrollo Social (CONEVAL, 2010), en el año 2008, México tenía 5.1 millones de niños y jóvenes viviendo en condiciones de pobreza extrema, otros 15.7 millones vivían en condiciones de pobreza moderada y 11.2 millones se encontraban en una situación de vulnerabilidad por carencia social. En números relativos, las cifras anteriores indican que para ese año $60 \%$ de los pobres en el país eran niños y jóvenes menores de 18 años, situación que evidencia la desigualdad, las condiciones de carencias, la falta de oportunidades, y en definitiva, permite observar la situación de vulnerabilidad en relación a los derechos en que se encuentra la infancia en México (Padrón \& González, 2012).
A esta dura y agobiante realidad de la pobreza presente, se suma la incertidumbre futura para el desarrollo de una trayectoria laboral en condiciones dignas y decentes, de niños y jóvenes que trabajan y no estudian. Esto es así, ya que se reconoce que las primeras inserciones en el mercado de trabajo, condicionan el desarrollo laboral de una persona. En este sentido, la promoción de trayectorias laborales positivas para la fuerza laboral de la población NNA está intrínsecamente relacionada con el acceso a un trabajo decente, tanto como con las posibilidades de mantenerse estudiando a la edad en que deberían hacerlo.

La inserción laboral a temprana edad (trabajo infantil y adolescente) es uno de los factores que al condicionar la permanencia en el sistema educativo de quienes realizan ese trabajo, en el largo plazo, afecta la acumulación de capital humano. Un proceso que trae aparejado, en la mayoría de los casos, itinerarios laborales deficientes que los lleva engrosar la masa de trabajadores no calificados $e$ insertos en actividades sumamente precarias, o a convertirse en personas en las fronteras de los circuitos sociales (grupo que no estudian, ni trabajan), o emprender la aventura de la emigración indocumentada (OIT, 2007).

\section{Las cifras del trabajo infantil en México}

De acuerdo con las estimaciones del MTI 2009, en México 3.014 .800 niñas y niños de 5 a 17 años ${ }^{10}$ realizaron alguna actividad económica durante el período de referencia ( $10,7 \%$ de la población total de referencia). De los poco más de 3 millones de niñas y niños ocupados, se estimó que aproximadamente 1.2 millones $(39,7 \%)$ no asistían a la escuela. Del total de niños y jóvenes que no asistían a la escuela, correspondían a este grupo de edad 48,6\% de hombres y $44,1 \%$ de mujeres. Dejar la escuela antes del tiempo establecido significa el riesgo de continuar el aprendizaje predominantemente en las calles. Con esto, las capacidades y oportunidades

\footnotetext{
10 Se hace referencia a este grupo de edad porque el Módulo de Trabajo Infantil define su población de interés entre estas edades, es decir, trabaja con niñas, niños y adolescentes entre 5 y 17 años.
} 
de los adolescentes se ven recortadas de manera drástica, y sus riesgos de salud aumentan. La mayoría de ellos además estarán condenados a vivir en situación de pobreza.

Son las localidades menos urbanizadas (las que tienen menos de 100 mil habitantes) las que presentan mayores niveles de trabajo infantil con 70,3\%, en contraposición con el $29,7 \%$ de niños y niñas ocupadas que residen en localidades con 100 mil habitantes o más.

En cuanto a las diferencias en términos geográficos, se observa que es el Estado de México el que concentró el mayor porcentaje de niños y niñas ocupadas en el país con 9,5 por ciento (cifra que representa 287 mil niños y niñas ocupadas). A este le siguen Puebla con 245 mil $(8,1 \%)$ y Jalisco con 240 mil (8,0\%). En el otro extremo se encuentran Baja California Sur, con poco menos de 12 mil niños y niñas ocupadas $(0,4 \%$ del total nacional), entidad federativa que reportó la cifra más baja de niños y niñas incorporados a actividades económicas, seguida por el Distrito Federal en donde 94 mil niñas y niños trabajaron ( $3,1 \%$ del trabajo infantil total).

Los datos indican que del total de niñas, niños y adolescentes ocupados (3.014.800), 28,4\% tenían entre 5 y 13 años de edad y 71,6\% tenían entre 14 y 17 años. En cuanto el sexo de la población infantil ocupada, los datos indican que $66,9 \%$ son hombres y $33,1 \%$ son mujeres.

La información secundaria ofrecida por la encuesta permite una aproximación a los efectos que tiene el trabajo infantil sobre sus hogares o grupos familiares, de esta manera se puede decir que 59,5\% de los niños y las niñas ocupadas trabajan para un familiar, 36,2\% para un no familiar y 4,3\% laboraban solos o por su cuenta (Tabla 1 ).
Tabla 1. Indicadores básicos de la población de NNA ocupada, MTI, ENOE; 2009 ${ }^{11}$

\begin{tabular}{|c|c|}
\hline & Porcentajes \\
\hline \multicolumn{2}{|l|}{ Personas para las que trabaja o ayuda } \\
\hline Familiar & 59.5 \\
\hline No familiar & 36.2 \\
\hline Trabaja solo o por su cuenta & 4.3 \\
\hline Total & 100.0 \\
\hline \multicolumn{2}{|l|}{ Condición de aportación de ingresos al hogar } \\
\hline Si aporta & 33.6 \\
\hline No aporta & 66.4 \\
\hline Total & 100.0 \\
\hline \multicolumn{2}{|l|}{ Motivos por los que trabaja } \\
\hline El hogar necesita de su aportación económica & 11.9 \\
\hline El hogar necesita de su trabajo & 28.7 \\
\hline Aprender un oficio & 20.2 \\
\hline Para pagar su escuela y/o sus propios gastos & 23.4 \\
\hline No quiere ir a la escuela & 3.9 \\
\hline Otra razón & 11.9 \\
\hline Total & 100.0 \\
\hline \multicolumn{2}{|l|}{ Consecuencias de dejar de trabajar } \\
\hline \multicolumn{2}{|l|}{ Hogares } \\
\hline Tendría que contratarse a alguien para que lo supliera & 5.0 \\
\hline El ingreso del hogar se vería afectado & 17.0 \\
\hline Otra consecuencia & 22.1 \\
\hline No habría consecuencias & 55.2 \\
\hline No especificado & 0.7 \\
\hline Total & 100.0 \\
\hline \multicolumn{2}{|l|}{ Personales } \\
\hline No habría dinero para su vestido y/o diversión & 30.0 \\
\hline No aprendería un oficio y/o se volvería irresponsable & 19.3 \\
\hline Volvería a la escuela o a los quehaceres del hogar & 2.8 \\
\hline Otra consecuencia & 5.6 \\
\hline No habría consecuencias & 41.4 \\
\hline No especificado & 0.9 \\
\hline Total & 100.0 \\
\hline
\end{tabular}

11 Fuente: Instituto Nacional de Estadística y Geografía, 2009. Encuesta Nacional de Ocupación y Empleo, Módulo de Trabajo Infantil. México. 
Se puede observar, a partir de la información proporcionada por la encuesta, que en $44,1 \%$ de los casos de NNA ocupados se tendría un efecto económico sobre el hogar si éstos dejaran de trabajar, ya sea porque se tendría que contratar a alguien $(5,0 \%)$, porque el ingreso del hogar se vería afectado $(17,0 \%)$, o tendría otra consecuencia $(22,1 \%)$. Es decir, que en poco menos de la mitad de los hogares si los niños o niñas dejaran de trabajar se tendría un efecto, contra el $55,0 \%$ donde se reporta que no se tendría ninguna consecuencia. Relacionado con esto, se tiene que los niños y niñas ocupadas que aportaron toda o una parte del ingreso a su hogar fueron 33,6\% del total.

Esta cifra obliga a revisar la perspectiva que desde el enfoque de las estrategias familiares de reproducción, postula el trabajo infantil precisamente como uno de los mecanismos mediante los cuales los hogares y las familias aseguran su sobrevivencia. Con la incorporación de NNA al mundo del trabajo no sólo se disminuye la carga laboral del adulto, sino que además, les posibilita obtener dinero, alimentos y vestimenta que todos los miembros de la familia comparten y que sirven para cubrir las necesidades más básicas del conjunto familiar o que, coadyuva a la familia si el NNA logra su auto-sostenimiento.

En esta dinámica, el trabajo de los niños, niñas y adolescentes se inserta dentro de una lógica de división del trabajo familiar. En el desarrollo de sus estrategias de reproducción, el grupo familiar se organiza siguiendo ciertas pautas de división del trabajo que se encuentran ligadas con valoraciones $y$ representaciones acerca de los roles que debe ocupar cada integrante (Rausky, 2009).

En cuanto a los motivos por los que trabajan los niños y las niñas se tiene que $11,9 \%$ lo hace por necesidades económicas del hogar, $28,7 \%$ porque el hogar necesita de su trabajo, 20,6\% por aprender un oficio y $23,4 \%$ para pagar los gastos de la escuela o los propios gastos del niño o niña. Con respecto a esto último, los estados que registraron las tasas de inasistencia escolar más altas de niños y niñas ocupadas fueron Chihuahua (62,3\%), Querétaro (51,7\%), Guanajuato $(49,1 \%)$, Chiapas (49\%) y Morelos (48,8\%).

Otro aspecto a resaltar sobre la información proporcionada por la encuesta, tiene que ver con las actividades domésticas realizadas por las niñas y niños, y que como se mencionó es importante de analizar ya que representa un factor poco estudiado pero que es de gran importancia para el desarrollo y el bienestar de la población NNA.

Es evidente la importancia de los hogares en la configuración del trabajo infantil. Por eso es que se debe resaltar que en 2009 México tenía en total 15.1 millones de hogares con niños y niñas de 5 a 17 años, en 2.4 millones del total de hogares referidos había niños y niñas ocupadas, esta cifra representa el 15,8\% del total de hogares con niños en estas edades. De acuerdo con las características del jefe o jefa del hogar, en 12,8\% de los hogares con niños de 5 a 17 años ocupados, el jefe del hogar no trabajó durante la semana de referencia; y en el 23,8\% de los hogares con niños ocupados en el grupo de edad manejado la unidad doméstica era jefaturada por una mujer. Cuando se trata de hogares con jefatura femenina, el trabajo de los niños, niñas y adolescentes adquiere otra relevancia, debido a que la intensidad y responsabilidad que asume en la realización del trabajo es mayor; ya no es vista como una colaboración más, sino que pasa a ser central y por lo tanto, adquiere mayor preponderancia (Rausky, 2009). 
Tabla 2. Indicadores básicos de los hogares con población de 5 a 17 años ocupada. MTI - ENOE, 200912

\begin{tabular}{|c|c|}
\hline Indicadores & Porcentajes \\
\hline \multicolumn{2}{|l|}{ Tipo y clase de hogar } \\
\hline Familiar & 99.5 \\
\hline Nuclear & 69.6 \\
\hline Jefe 0 jefa con hijos & 17.7 \\
\hline Pareja con o sin hijos & 82.3 \\
\hline No nuclear & 30.4 \\
\hline No familiar & 0.5 \\
\hline Total & 100.0 \\
\hline \multicolumn{2}{|l|}{ Tamaño del hogar } \\
\hline De 1 a 3 integrantes & 14.5 \\
\hline De 4 a 5 integrantes & 45.0 \\
\hline De 6 a 7 integrantes & 26.6 \\
\hline 8 integrantes o más & 13.9 \\
\hline Total & 100.0 \\
\hline \multicolumn{2}{|l|}{ Nivel de instrucción del jefe } \\
\hline Sin instrucción & 14.1 \\
\hline Primaria incompleta & 24.6 \\
\hline Primaria completa & 25.5 \\
\hline Secundaria incompleta & 3.8 \\
\hline Secundaria completa & 20.0 \\
\hline Algún año de preparatoria o más & 12.0 \\
\hline Total & 100.0 \\
\hline \multicolumn{2}{|l|}{ Condición de ocupación del jefe } \\
\hline Ocupado & 87.2 \\
\hline No ocupado & 12.8 \\
\hline Total & 100.0 \\
\hline
\end{tabular}

Los datos evidencian que existe una relación entre el nivel de instrucción del jefe y la proporción de hogares con niños ocupados, es decir que, en los hogares donde el jefe tiene menores niveles de instrucción el porcentaje de niños ocupados es mayor; en contraposición, en aquellos hogares con jefes de mayor escolaridad, el porcentaje de hogares con niños ocupados es menor. En resumen, en 30,1\% de los hogares donde el jefe no tiene instrucción hay niños ocupados, en contraste con el 7,5\% de los hogares donde el jefe tenía algún año de preparatoria o más.
A partir de esta presentación general de los datos provenientes del MTI 2009 es posible extraer algunas líneas de análisis que resultan importantes aunque, como quedó explicitado, el tema del trabajo infantil es por demás complejo, y por lo tanto, requiere de un estudio más profundo que una aproximación diagnóstica como es el caso de este trabajo.

Una de estas dimensiones tiene que ver con que el trabajo infantil representa una contribución importante para la reproducción del hogar, no sólo porque fomenta la cooperación y la solidaridad entre los miembros de la unidad doméstica, ayudando a realizar actividades y tareas necesarias, sino también porque permite a la unidad familiar obtener ingresos, acceder a bienes y servicios, y facilita la incorporación de otros miembros del hogar a una actividad económica.

Sin embargo, no se puede perder de vista que en la mayoría de los casos el trabajo infantil es una dimensión que tiene impactos importantes sobre la escolaridad de los niños y niñas, siendo causa de abandono escolar y de una escasa formación en capital humano, como se menciona anteriormente, factores que contribuyen a perpetuar la pobreza y a limitar el desarrollo a largo plazo, con todo lo que implica en términos de inclusión y del ejercicio efectivo de una ciudadanía integrada e integral.

En el corto plazo, si bien el trabajo infantil puede contribuir a incrementar el ingreso familiar, a que éste se mantenga o que no disminuya de forma drástica; en el largo plazo favorece el aumento de la desigualdad en la distribución del ingreso, haciendo más grande la brecha que separa a los pobres y a los ricos, restringiendo las oportunidades para salir de la pobreza, ya que la escasa formación de recursos humanos y la baja acumulación de capital humano en el largo plazo inciden de manera negativa en el nivel educativo de la población, la productividad de la fuerza laboral y su competitividad.

\section{A modo de reflexiones finales}

A partir de este breve recorrido hemos intentado dar cuenta del lugar que tiene el trabajo de niños, niñas $y$ adolescentes en la realidad de México, desde una

12 Fuente: Instituto Nacional de Estadística y Geografía, 2009. Encuesta Nacional de Ocupación y Empleo, Módulo de Trabajo Infantil. México. 
aproximación conceptual y empírica del fenómeno. Los datos han puesto de manifiesto que los NNA que trabajan en su mayoría no estudian, que las zonas rurales son las que presentan una mayor incidencia de este fenómeno, que hay diferencias regionales que posiciona al Estado de México como la entidad en la que se registran mayor cantidad de casos de trabajo infantil y que la mayoría de los NNA trabajan para un familiar. El hecho de que las prácticas laborales se desarrollen en contextos familiares tiñe a dichas prácticas de un componente afectivo que redefine los contenidos mismos del trabajo. A diferencia de las distancias sociales y afectivas que caracterizan al trabajo en las modernas sociedades urbanas, se vislumbran pautas de interacción dotadas de un importante componente afectivo-emocional como son la confianza, el compromiso y la colaboración mutua (Rausky, 2009) lo que tiene incidencia en el destino de los ingresos derivados de esa actividad.

El trabajo infantil como mecanismo de sobrevivencia de la familia, también ha sido puesto en discusión con la lectura de los datos, en tanto por un lado parece ser demasiado simple el enfoque que sitúa a las estrategias de sobrevivencia desde una perspectiva que caracteriza la adecuación de las formas de organización familiar a la satisfacción de necesidades en períodos económicos críticos. Los estudios sobre estrategias de sobrevivencia tienden a pensar estas estrategias como coyunturales en función a situaciones de emergencia, el trabajo infantil y otras manifestaciones de estas estrategias habrían de desaparecer por tanto, tan pronto se supera la condición de coyuntura (Maureira, 2002). Por otra parte, los datos han reflejado que los ingresos provenientes del trabajo de niños, niñas y adolescentes contribuyen al hogar del que forman parte aún si no es aportado directamente al hogar, mediante el autosostenimiento del menor.

La regulación del trabajo de niños, niñas y adolescentes se ubica también como un problema complejo, desde las definiciones del fenómeno, pasando por las formas de medición y atravesando la complicada encrucijada de las percepciones y valoraciones sociales, colectivas y familiares del trabajo de NNA.

En la atención y acompañamiento a los niños y las niñas que viven situaciones como las descritas, que en muchos casos implican violencia, vulnerabilidad, exclusión y el no ejercicio de una serie de derechos, se pueden mencionar tres retos principales:

- Identidad: en el reconocimiento de este grupo de población como sujetos capaces de participar en el mejoramiento de sus condiciones de vida y no como objetos a los que hay que utilizar, manipular o en el peor de los casos, negar. Reconocer un rostro y un corazón al cual dotar de sabiduría y firmeza en vez de números para llenar estadísticas oficiales y justificar recursos.

- Seguridad: en el reconocimiento de sus modos de organización y participación y los de los grupos, colectivos y organizaciones presentes en su entorno a los cuales se debe fortalecer. Reconocer la voluntad y capacidad de miles de personas por participar en el mejoramiento de sus condiciones de vida en lugar de mantener los esfuerzos y recursos nunca suficientes para intentarlo lograr desde las instituciones y organizaciones oficiales.

- Sostenibilidad: en el reconocimiento de otros ámbitos diferentes al económico que influyen en las condiciones de vida de la gente, la dinámica de fuerzas que interactúan en las comunidades y grupos humanos y que, positivamente, tanta riqueza dan a éstos.

Estos retos van de la mano del reconocimiento de que en México no existe un diagnóstico integral $e$ interdisciplinario del trabajo infantil. Aunque se han realizado algunos estudios desde diversas disciplinas -sociología, economía, demografía, derecho- es necesario profundizar en el tema a través de un diálogo entre las distintas visiones. A partir de la inclusión del MTI en la ENOE se conocen otras dimensiones del problema y algunos datos adicionales sobre el mismo. El siguiente paso debe ser una investigación que permita hacer un diagnóstico sobre las causas del problema, así como de las consecuencias del mismo. Esto supone comprender por qué los niños y niñas trabajan, para así desarrollar políticas públicas encaminadas a la atención integral y holística del fenómeno y la erradicación de la explotación.

Es de reconocer que analizar el trabajo infantil implica abordar un fenómeno de naturaleza extremadamente compleja, y que muchas veces se ve invisi- 
bilizado por desarrollarse en el ámbito privado (por ejemplo, el trabajo doméstico en el propio hogar), o "cuasi-privado" (por ejemplo, el trabajo desarrollado en un negocio familiar). En este sentido la fuente de información imprime una dificultad adicional, ya que las actividades que se buscan analizar son intermitentes, poco visibles, muchas veces no consideradas como trabajo y por lo tanto, poco valoradas y reconocidas (Padrón \& Navarrete, 2012).

\section{Referencias}

Alzate, M. V. (2002) "Concepciones e imágenes de la infancia". En: Revista de Ciencias Humanas, 8 (28). Universidad Tecnológica de Pereira. Colombia.

Barrientos, C. \& Corvalán, N. (1996) "Cosas de niños. Investigación de la experiencia histórica infantil en los procesos de modernización. Notas de discusión". En: Última década. № 004, Centro de Investigación y Difusión Poblaciones de Achupallas. Chile.

Cámara de Diputados del H. Congreso de la Unión (2013, Junio) "Ley Federal del Trabajo". "Ley Federal del Trabajo". Disponible en: http://www.diputados.gob.mx/LeyesBiblio/pdf/125.pdf

Cámara de Diputados del H. Congreso de la Unión (2013, Junio) "Constitución mexicana". Constitución Política de los Estados Unidos Mexicanos. Disponible en: http://prodecon.gob.mx/ Documentos/MarcoNormativo/1-ConstitucionPolitica.pdf

CONEVAL (2013, Junio) "Consejo Nacional de Evaluación de la Política de Desarrollo Social”. Análisis y medición de la pobreza. Disponible en: http://www.coneval.gob.mx/medicion/ Paginas/Medici\%C3\%B3n/Pobreza-2010.aspx

González Contró, M. (2011) Derecho de familia en México. Instituto de Investigaciones Jurídicas, UNAM, México.

González Contró, M. (2011) “'Menores o niñas, niños y adolescentes? Reflexiones en el contexto del debate en América Latina”. En: Pérez Contreras, M. \& Macías Vázquez, M. (Coord.). Marco Teórico Conceptual sobre Menores versus Niñas, Niños y Adolescentes. Instituto de Investigaciones Jurídicas - UNAM. México.

González, M. (2008) Derechos humanos de los niños: una propuesta de fundamentación. IIJ-UNAM, México, D.F.

INEGI (2013) Censos Generales de Población y Vivienda 1950, 1960, 1970, 1980, 1990, 2000 y 2010. VII, VIII, IX, X, XI y XII. INEGI. I y II Conteos de Población y Vivienda 1995 y 2005.INEGI. II Conteo de Población y Vivienda, 2005. Consulta Interactiva de Datos Disponible en http://www.inegi.org.mx/

INEGI (2009) Documento sobre la Encuesta Nacional de Ocupación y Empleo. INEGI. México.

Kurczyn, P. (2006) Derechos del Pueblo Mexicano, Porrúa-Instituto de Investigaciones Jurídicas, Colección México a través de sus Constituciones, T. XX.

Maccise, M. (2006) Niños y niñas en situación de calle y la discriminación en el acceso a la educación, salud y justicia. Editado por Dirección General Adjunta de Estudios, Legislación y Políticas Públicas Consejo Nacional para Prevenir la Discriminación.
Colección de estudios y documentos de trabajo, D.F., México. Maureira, F. (2002) "Trabajo infantil. Algunas consideraciones desde la antropología”. En: Revista Austral de Ciencias Sociales, No. 6. Chile.

MTI- Módulo de Trabajo Infantil (2009) Documento metodológico. Instituto Nacional de Estadística y Geografía, México.

Naciones Unidas (2013, Junio) “CRC/C/MEX/CO/3”. Observaciones finales emitidas por el Comité de los Derechos del Niño de la Organización de las Naciones Unidas, respecto al III Informe de México sobre Niñez. Disponible en: http://www.unicef. org/mexico/spanish/mx_resources_informe_crc_mexico.pdf

Oficina del Alto Comisionado de las Naciones Unidas para los Derechos Humanos (2013, Junio) "Convención sobre los Derechos del Niño". Convención sobre los Derechos del Niño. Disponible en: http://www2.ohchr.org/spanish/law/crc.htm

OIT (2013, Junio) "Convenio 138". Convenio sobre la edad mínima de admisión al empleo. Disponible en: http://www. unicef.org/mexico/spanish/mx_resources_138_OIT.pdf

OIT (2013, Junio) “Convenio 182". Convenio sobre la Prohibición de las Peores Formas de Trabajo Infantil y la Acción Inmediata para su Eliminación. Disponible en: http://www.pgjdf.gob. $\mathrm{mx} /$ temas/4-6-1/fuentes/5-a-16.pdf

OIT (1996) El trabajo infantil. Lo intolerable en el punto de mira. Ginebra.

OIT (2007) Trabajo decente y juventud en América Latina. Lima, Perú: Oficina Regional para América Latina y el Caribe.

Padrón, M. \& González, M. (2012) "Encuadre normativo y empírico para un diagnóstico del trabajo infantil en México". En: Oñati Socio-Legal Series, Vol. 2, (2). Cooperatives and Collective Enterprise in the Social Economy, España.

Padrón, M. \& Román, P. (2010) "La infancia como unidad de análisis en la investigación social. Problema actual y desafío para el futuro". En: González Contró, M. (coordinadora) Los derechos de niños, niñas y adolescentes en México a 20 años de la Convención sobre los derechos del niño, IIJ-UNAM; México.

Padrón, M. (2012) "Análisis de la participación laboral de la población de 12 a 17 años en México, con un enfoque de derechos". Ponencia presentada en la XI Reunión Nacional de Investigación Demográfica en México. Cambios demográficos y políticas públicas, realizada del 30 de mayo al 1 de junio de 2012 en Aguascalientes, Aguascalientes, México.

Padrón, M. \& Navarrete, E. (2012), "Una mirada sobre el trabajo infantil en México. El Modulo de Trabajo Infantil de la ENOE". En: Coyuntura Demográfica, $\mathrm{N}^{\circ} 2$. México.

Rausky, M. E. (2009) "Trabajo y familia: el aporte de los niños trabajadores a la reproducción del hogar". En: Trabajo y Sociedad, №. 12. Santiago del Estero, Argentina.

Salazar, M. (1996) "Explotación económica y educación del niño en América Latina". En: Verdugo, Miguel Ángel \& Soler-Sala, Víctor (ed.) La Convención de los Derechos del Niño. Hacia el siglo XXI, Universidad de Salamanca.

Secretaría del Trabajo y Previsión Social (2013, Junio) Reglamento Interior de la Secretaría del Trabajo y Previsión Social. "REGLAMENTO Interior de la Secretaría del Trabajo y Previsión Social. Disponible en: http://www.stps.gob.mx/bp/anexos/ reglamento_interior.pdf

Vázquez, R. (2007) Teoría del derecho. Oxford University Press, México. 